\title{
Two-Sided Matching Decision-Making with Uncertain Information Under Multiple States
}

\author{
Shengqun CHEN \\ School of Electronic Information Science, Fujian Jiangxia University, Fuzhou 350108, China; \\ Decision Sciences Institute, Fuzhou University, Fuzhou 350116, China \\ E-mail: csq255@qq.com \\ Yingming WANG \\ Decision Sciences Institute, Fuzhou University, Fuzhou 350116, China \\ E-mail:msymwang@hotmail.com \\ Hailiu SHI \\ School of Electronic Information Science, Fujian Jiangxia University, Fuzhou 350108, China; \\ Decision Sciences Institute, Fuzhou University, Fuzhou 350116, China \\ E-mail: 627498695@qq.com \\ Yang LIN \\ Decision Sciences Institute, Fuzhou University, Fuzhou 350116, China \\ E-mail: linyang42@163.com \\ Meijuan LI \\ School of Economics and Management, Fuzhou University, Fuzhou 350116, China \\ E-mail: 758300@qq.com
}

\begin{abstract}
A novel decision-making method based on evidential reasoning is proposed for solving the two-sided matching problem with uncertain information under multiple states in this paper. Firstly, the discernment frame of evidence is constructed for two-sided matching. Secondly, the preference ordinal values given by two-sided decision-makers are transformed into rank belief degrees. On this basis, and with two-sided satisfaction as the goal, two-sided rank belief degrees are taken as pieces of evidence, and satisfaction degrees of two-sided matching are obtained through evidence fusion. Then, a decision-making model based on satisfaction degrees is constructed in order to obtain the matching solution. Finally, an illustrative example demonstrates the application of the proposed approach.
\end{abstract}

Keywords matching decision-making; uncertain information; multiple states; evidence fusion

Received August 9, 2015, accepted January 7, 2016

Supported by the National Natural Science Foundation of China Project (71371053, 71403055), Fujian Provincial Natural Science Foundation Project (2015J01279), and Fujian Province Young Teachers' Education Scientific Research Project (JA14322) 


\section{Introduction}

Two-sided matching markets are typically characterized as consisting of two kinds of heterogeneous agents, each of whom have preferences regarding potential matches with agents of the other kind; that is, both sides make decisions and rank the opposite side using a matching procedure. For example, hospitals are careful about the doctors they hire, while doctors prefer to work at certain hospitals. Two-sided matching was first introduced by Gale and Shapley ${ }^{[1]}$ and later extended and refined by other contributors. Therefore, it is also known as the twosided matching theory, or matching theory, for short. The matching theory can achieve an efficient outcome that advances the common interests or reconciles the conflicting interests of agents wishing to be matched. As result, it has been applied to a wide range of areas including schooling choices ${ }^{[2,3]}$, employer-employee job matching ${ }^{[4,5]}$, mergers and acquisitions ${ }^{[6-8]}$, intercompany matching ${ }^{[9]}$, choices regarding bank lenders ${ }^{[10]}$, assignment of CEOs to firms ${ }^{[11,12]}$, buyer-supplier relationships $^{[13,14]}$, firm-university relationships ${ }^{[15]}$, etc.

While the matching theory has a long and rich history, much of the existing literature assumes complete information; most studies assume that all agents fully know their own preferences. In fact, incomplete information is arguably commonplace in most environments. Thus, solving matching problems under uncertainty needs a novel approach. The matching theory under uncertainty was first developed by Roth ${ }^{[16]}$ and later extended and improved by other scholars $^{[17-20]}$. Notably, recent years have recorded increasing interest in this area. Rastegari, et al. ${ }^{[21]}$ proposed a novel model of two-sided matching in which agents are endowed with known partially ordered preferences and unknown true preferences drawn from known distributions consistent with the partial order. Chade, Lewis and Smith ${ }^{[22]}$ devised an equilibrium model of college admissions that analyzes the impact of two previously unexplored frictions in the application process: students find it costly to apply to colleges, while it is uncertain how colleges may evaluate their applications. Liu, et al. ${ }^{[23]}$ formulated the notion of stable outcomes in matching problems with one-sided asymmetric information and provided sufficient conditions for efficient incomplete information stable matching. In addition, two-sided matching with uncertainty was also studied by Chen, Fan and $\mathrm{Li}^{[25]}$, Yue ${ }^{[26-28]}$, Liang and Jiang ${ }^{[29,30]}$, Zhang and $\mathrm{Hu}^{[24]}$, and Chen, Wang and Shi ${ }^{[31,32]}$.

Existing matching models (or matching decision-making methods) have made significant headway in solving matching problems with different uncertain assessment information. Unfortunately, almost all these research results fail to take uncertainty under different states into account. In reality, agents often face multiple future states when they rank the opposite side in a matching procedure. Each of them may have different preferences over potential matches with agents on the other side under different states. For example, under state $\theta_{1}$, agent $A$ ranks agent $B$ of the opposite side in the first place. Under state $\theta_{2}, A$ ranks $B$ in the third place. Under state $\theta_{3}, A$ does not rank $B$ at all. Suppose that the probabilities of states $\theta_{1}, \theta_{2}, \theta_{3}$ occurring are $0.5,0.3$, and 0.2 , respectively. Thus, $A$ 's preference values toward $B$ are distributive belief degrees $\{(1,0.5) ;(3,0.3) ;(H, 0.2)\}$, with $H$ indicating the unknown preference. As different types of data have different requirements for decision-making methods, it is difficult to directly apply existing methods to solve two-sided matching problems with uncertain information under multiple states. This paper thus explores a matching method based on the evidential reasoning 
(ER) approach ${ }^{[33,34]}$, which is based on the decision theory and the Dempster-Shafer (D-S) theory of evidence ${ }^{[35,36]}$, and it can be used to model various uncertainties in the matching process.

This paper is organized as follows. Section 2 develops an evidence fusion method for matching decision-making problems with uncertain information under multiple states. Section 3 provides a practical example to illustrate the proposed methodology. Finally, Section 4 concludes the paper.

\section{Evidence Fusion Method}

Our basic reasoning in this paper takes two-sided matching satisfaction as the main goal and uncertain preference information of matching parties $A$ and $B$ under various states as target evidence. We then construct an evidence fusion model and take the two-sided fusion results obtained therein as the two-sided matching satisfaction degrees. Finally, we use a 0-1 assignment model to obtain a two-sided matching solution.

\subsection{Constructing Unified Assessment Grades on Both Sides}

Let the matching agents on one side be $A_{1}, A_{2}, \cdots, A_{P}$ (party $A$ ) and those on the other side be $B_{1}, B_{2}, \cdots, B_{Q}$ (party $B$ ). In order to use the ER approach to conduct decision analysis for the matching problem, it is necessary to define a unified evidence frame of discernment for the two-sided matching agents. Suppose all the agents on both sides are assessed using $N$ crisp assessment grades $H_{n}(n=1,2, \cdots, N)$, which are mutually exclusive and collectively exhaustive. The $N$ assessment grades form the frame of discernment $H=\left\{H_{1}, H_{2}, \cdots, H_{N}\right\}$ in the ER approach.

Let $Y_{n}(n=1,2, \cdots, N)$ be the standard values of assessment grades $H_{n}$. For party $A$, let the standard values of the assessment grades be defined as follows:

$$
Y_{1}=1, \quad Y_{2}=1+\frac{P-1}{N-1}, \quad Y_{3}=1+2 \frac{P-1}{N-1}, \quad \cdots, \quad Y_{N}=1+(N-1) \frac{P-1}{N-1} .
$$

For party $B$, let the standard values of assessment grades be defined as follows:

$$
Y_{1}=1, \quad Y_{2}=1+\frac{Q-1}{N-1}, \quad Y_{3}=1+2 \frac{Q-1}{N-1}, \cdots, \quad Y_{N}=1+(N-1) \frac{Q-1}{N-1} .
$$

In Equations (1) and (2), the standard values of assessment grades $Y_{n}(n=1,2, \cdots, N)$ are equal to the relative positions on both sides. For instance, suppose $P=9$ and $Q=5$, and we use five crisp assessment grades. Then, the calculated results of the standard values of party $A$ are $1,3,5,7$, and 9 according to Equation (1), while those of party $B$ are 1, 2, 3, 4, and 5 according to Equation (2). Clearly, for matching party $A$, the relative position with the highest value will be ranked first; the lowest value, ninth; and the middle value, fifth. For matching party $B$, the relative position with the highest value will be ranked first; the lowest value, fifth; and the middle value, third. It follows that a person ranked in the fifth place among a group of nine people and a person ranked in the third place among a group of five people will occupy the same relative position; thus, we ascertain that using relative positions to determine the standard values of two-sided evaluation of rankings is reasonable.

In order to model quantitative agents using the defined assessment grades, grade utilities need to be known. The utilities for each assessment grade can be elicited from decision-makers 
in different ways, but in this paper, we suppose that the utilities denoted by $u\left(H_{n}\right)(n=$ $1,2, \cdots, N)$ are set to

$$
u\left(H_{1}\right)=1, \quad u\left(H_{2}\right)=(N-2) /(N-1), \quad u\left(H_{3}\right)=(N-3) /(N-1), \cdots, \quad u\left(H_{N}\right)=0 .
$$

\subsection{Transforming Preference Ordinals into Belief Degrees of Evidence}

To use the ER approach to conduct decision analysis for matching problems, preference ordinals on both sides need to be transformed into belief degrees. Depending on three different types of data relationships between the preference ordinals and the standard values of the assessment grades, any preference ordinal can be transformed into a belief degree of the assessment grades.

In the first type of data relationship, preference ordinal $y_{i}$ happens to be equivalent to a certain standard value of assessment grade $Y_{n}(n=1,2, \cdots, N)$. Then, value $y_{i}$ can be equivalently expressed as the following belief degree:

$$
y_{i} \Leftrightarrow\left\{\left(H_{n}, \beta_{n}\right)\right\}
$$

where the symbol " $\Leftrightarrow$ " represents "is equivalent to", and $\beta_{n}=1$. This means that preference ordinal $y_{i}$ is equivalent to $Y_{n}$ with probability 100\%. In Equation (3), $\beta_{n}$ represents the belief degree of $H_{n}$.

In the second type of data relationship, preference ordinal $y_{i}$ lies between two adjacent assessment grades $H_{n}, H_{n+1}(n=1,2, \cdots, N-1)$, that is, $Y_{n}<y_{i}<Y_{n+1}$. For

$$
y_{i}=\frac{Y_{n+1}-y_{i}}{Y_{n+1}-Y_{n}} \cdot Y_{n}+\frac{y_{i}-Y_{n}}{Y_{n+1}-Y_{n}} \cdot Y_{n+1},
$$

value $y_{i}$ can be equivalently expressed as the following belief degree:

$$
y_{i} \Leftrightarrow\left\{\left(H_{n}, \beta_{n}\right) ;\left(H_{n+1}, \beta_{n+1}\right)\right\}
$$

where

$$
\beta_{n}=\frac{Y_{n+1}-y_{i}}{Y_{n+1}-Y_{n}}, \quad \beta_{n+1}=\frac{y_{i}-Y_{n}}{Y_{n+1}-Y_{n}} .
$$

This means that preference ordinal $y_{i}$ is either equivalent to $Y_{n}$ with probability $\beta_{n}$ or equivalent to $Y_{n}$ with probability $\beta_{n+1}$. Note that $\beta_{n}+\beta_{n+1}=1$.

In the third type of data relationship, preference ordinal $y_{i}$ is totally unknown. Thus, value $y_{i}$ can be equivalently expressed as the following belief degree:

$$
y_{i} \Leftrightarrow\left\{\left(H, \beta_{H}\right)\right\},
$$

where $H=\left\{H_{1}, H_{2}, \cdots, H_{N}\right\}$ and $\beta_{H}=1$. This means that preference ordinal $y_{i}$ must lie at a certain assessment grade, but its specific location is unknown.

According to Equations (3) (5), all the preference ordinal values of two-sided matching agents under various states can be transformed into belief degrees.

\subsection{Determining the Degree of Satisfaction Between Two-Sided Matching Agents}

Taking the evaluation values of two-sided matching agents under various states as evidence of the degree of satisfaction, and then using $\mathrm{ER}^{[28]}$ for evidence fusion, we can determine the satisfaction degree of the two-sided matching. The process is briefly described in the following steps. 
The first step is to transform the original belief degrees into basic probability masses by combining the relative weights using the following equations:

$$
\begin{cases}m_{i}\left(H_{n}\right)=w_{i} \beta_{n, i}, & i=1,2, \cdots, L ; n=1,2, \cdots, N \\ \widetilde{m}_{i}(H)=w_{i}\left(1-\sum_{n=1}^{N} \beta_{n, i}\right), & i=1,2, \cdots, L \\ \bar{m}_{i}(H)=1-w_{i}, & i=1,2, \cdots, L \\ m_{i}(H)=\bar{m}_{i}(H)+\widetilde{m}_{i}(H), & i=1,2, \cdots, L\end{cases}
$$

with $\sum_{i=1}^{L} w_{i}=1$. Note that $m_{i}(H)$, which is not assigned to any assessment grade, contains two parts, $\bar{m}_{i}(H)$ and $\tilde{m}_{i}(H)$, where $\bar{m}_{i}(H)$ is caused by the relative importance of the $i^{\text {th }}$ evidence, and $\widetilde{m}_{i}(H)$, by the incompleteness of the evaluation.

The second step is to aggregate all pieces of evidence by combining the basic probability masses generated above. All pieces of evidence are combined using the following equations:

$$
\left\{\begin{array}{l}
m_{1} \oplus m_{2} \cdots \oplus m_{L}\left(H_{n}\right)=\frac{\sum_{A_{1} \cap A_{2} \cdots \cap A_{L}=H_{n}, A_{1}, A_{2}, \cdots, A_{L} \subseteq H} m_{1}\left(A_{1}\right) m_{2}\left(A_{2}\right) \cdots m_{L}\left(A_{L}\right)}{\sum_{A_{1} \cap A_{2} \cdots \cap A_{L} \neq \emptyset, A_{1}, A_{2}, \cdots, A_{L} \subseteq H} m_{1}\left(A_{1}\right) m_{2}\left(A_{2}\right) \cdots m_{L}\left(A_{L}\right)} \\
\bar{m}_{1} \oplus \bar{m}_{2} \cdots \oplus \bar{m}_{L}(H)=\frac{\bar{m}_{1}(H) \bar{m}_{2}(H) \cdots \bar{m}_{L}(H)}{\sum_{A_{1} \cap A_{2} \cdots \cap A_{L} \neq \emptyset, A_{1}, A_{2}, \cdots, A_{L} \subseteq H} m_{1}\left(A_{1}\right) m_{2}\left(A_{2}\right) \cdots m_{L}\left(A_{L}\right)} .
\end{array}\right.
$$

The third step is to normalize the aggregated basic probability masses into overall belief degrees using the following equations:

$$
\left\{\begin{array}{l}
\beta_{n}=\frac{m_{1} \oplus m_{2} \cdots \oplus m_{L}\left(H_{n}\right)}{1-\left(\bar{m}_{1} \oplus \bar{m}_{2} \cdots \oplus \bar{m}_{L}(H)\right)}, \quad H_{n} \subset H \\
\beta_{H}=\frac{\widetilde{m}_{1} \oplus \widetilde{m}_{2} \cdots \oplus \widetilde{m}_{L}(H)}{1-\left(\bar{m}_{1} \oplus \bar{m}_{2} \cdots \oplus \bar{m}_{L}(H)\right)}
\end{array}\right.
$$

where $\beta_{n}$ and $\beta_{H}$ are the belief degrees of the aggregated assessment. Note that the belief degree $\beta_{H}$ may be assigned to any assessment grade.

The final step is to determine the degree of satisfaction using the following equations:

$$
f\left(\beta_{1}, \beta_{2}, \cdots, \beta_{N}, \beta_{H}\right)=\sum_{n=1}^{N}\left[\beta_{n} \cdot u\left(H_{n}\right)\right]+\beta_{H} \cdot u\left(H_{H}\right) .
$$

When $\beta_{H}$ is assigned to the most preferred assessment grade $H_{1}$, the degree of satisfaction $f$ achieves its maximum, which is defined by Equation (10). However, when it is assigned to the least preferred assessment grade $H_{1}$, the degree of satisfaction $f$ achieves its minimum, which is defined by Equation (11). So, $f$ is, in fact, an interval if $\beta_{H} \neq 1$. Without loss of generality, we can use the midpoint of the interval, which is expressed as Equation (12), to determine $f$.

$$
\begin{aligned}
f_{\max } & =\sum_{n=1}^{N}\left[\beta_{n} \cdot u\left(H_{n}\right)\right]+\beta_{H} \cdot u\left(H_{1}\right) . \\
f_{\min } & =\sum_{n=1}^{N}\left[\beta_{n} \cdot u\left(H_{n}\right)\right]+\beta_{H} \cdot u\left(H_{N}\right) . \\
f_{\text {ave }} & =\left(f_{\max }+f_{\min }\right) / 2 .
\end{aligned}
$$




\subsection{Constructing the Two-Sided Matching Model}

Using the above-mentioned steps to find the average degree of satisfaction $f_{i j}$ of agent $A_{i}(i=1,2, \cdots, P)$ in party $A$ and $B_{j}(j=1,2, \cdots, Q)$ in party $B$, we can construct a twosided matching model as seen below:

$$
\begin{aligned}
\max & Z=\sum_{i=1}^{P} \sum_{j=1}^{Q} f_{i j} x_{i j} \\
\text { s.t. } & \sum_{j=1}^{Q} x_{i j}=1, \quad i=1,2, \cdots, P, \\
& \quad \sum_{i=1}^{P} x_{i j} \leq 1, \quad j=1,2, \cdots, Q, \\
& x_{i j}=0,1, \quad i=1,2, \cdots, P ; j=1,2, \cdots, Q .
\end{aligned}
$$

Equation (13) is a target function, enabling the greatest possible degree of satisfaction between the two-sided agents. Equation (14) is a constraining condition, indicating that each agent in party $A$ can only match with one other agent in party $B$. Equation (15) is a constraining condition, indicating that each agent in party $B$ matches with no more than one agent in party $A$. Equation (16) is a constraining condition, indicating that $x_{i j}=1$ when there is successful match between $A_{i}$ and $B_{j}$, and $x_{i j}=0$ when there is a match failure. This model is a standard 0-1 assignment model and can be solved using software such as Lingo or Cplex.

\section{Illustration}

Solving matching problems to select a business partner is a task of critical importance in supply chain management, where suppliers and retailers must find suitable business partners in order to form effective strategic alliances. Nine existing supply companies $\left(A_{1}, A_{2}, A_{3}, A_{4}, A_{5}\right.$, $\left.A_{6}, A_{7}, A_{8}, A_{9}\right)$ provide an assortment of different products. They receive cooperation offers from five retail companies $\left(B_{1}, B_{2}, B_{3}, B_{4}, B_{5}\right)$. According to market surveys, product sales are related to the current supply and sales of other products. There are three types of natural states \{ample supply of other products, average supply of other products, and insufficient supply of other products $\}$, represented by $\left\{\theta_{1}, \theta_{2}, \theta_{3}\right\}$. Under different natural states, retail companies will consider supply companies based on product quality, wholesale price, service, and other similar criteria. Using this evaluation, they put forth a preference order, as seen in Table 1. At the same time, the uncertainty in capital turnover causes retail companies to also have two different types of natural states \{prompt payment, delayed payment\}, represented by $\left\{\theta_{1}, \theta_{2}\right\}$. Under different natural states, suppliers also consider retail companies based on corporate reputation, cooperation experience, and other factors, which also results in a preference order, as seen in Table 2 . The data in the tables contain probability values for natural state occurrence and preference ordinal values; for example, in Table $1, A_{1}$ 's preference value for $B_{1}\{(1,0.5)$; $(-, 0.5)\}$ indicates that the probability of the first natural state occurring is 0.5 , and in this scenario, $A_{1}$ will rank $B_{1}$ in first place. The probability of the second scenario occurring is also 0.5, wherein $A_{1}$ will not rank $B_{1}$ among its preferences (indicated by “-"). 
Table 1 Suppliers' uncertain preference ordinal values for retailers under different states

\begin{tabular}{cccccc}
\hline & $B_{1}$ & $B_{2}$ & $B_{3}$ & $A_{4}$ & $A_{5}$ \\
\hline$A_{1}$ & $(1,0.5) ;(-, 0.5)$ & $(-, 0.4) ;(-, 0.6)$ & $(2,0.6) ;(3,0.4)$ & $(4,0.7) ;(5,0.3)$ & $(1,0.2) ;(3,0.8)$ \\
$A_{2}$ & $(2,0.5) ;(-, 0.5)$ & $(2,0.4) ;(-, 0.6)$ & $(1,0.6) ;(3,0.4)$ & $(1,0.7) ;(3,0.3)$ & $(2,0.2) ;(-, 0.8)$ \\
$A_{3}$ & $(3,0.5) ;(4,0.5)$ & $(1,0.4) ;(2,0.6)$ & $(-, 0.6) ;(5,0.4)$ & $(2,0.7) ;(-, 0.3)$ & $(3,0.2) ;(4,0.8)$ \\
$A_{4}$ & $(-, 0.5) ;(5,0.5)$ & $(3,0.4) ;(4,0.6)$ & $(3,0.6) ;(4,0.4)$ & $(3,0.7) ;(4,0.3)$ & $(-, 0.2) ;(5,0.8)$ \\
$A_{5}$ & $(2,0.5) ;(3,0.5)$ & $(4,0.4) ;(5,0.6)$ & $(-, 0.6) ;(5,0.4)$ & $(-, 0.7) ;(5,0.3)$ & $(2,0.2) ;(3,0.8)$ \\
$A_{6}$ & $(1,0.5) ;(-, 0.5)$ & $(3,0.4) ;(4,0.6)$ & $(2,0.6) ;(-, 0.4)$ & $(1,0.7) ;(-, 0.3)$ & $(1,0.2) ;(-, 0.8)$ \\
$A_{7}$ & $(2,0.5) ;(-, 0.5)$ & $(2,0.4) ;(-, 0.6)$ & $(1,0.6) ;(-, 0.4)$ & $(2,0.7) ;(-, 0.3)$ & $(2,0.2) ;(-, 0.8)$ \\
$A_{8}$ & $(2,0.5) ;(3,0.5)$ & $(1,0.4) ;(2,0.6)$ & $(4,0.6) ;(5,0.4)$ & $(2,0.7) ;(3,0.3)$ & $(2,0.2) ;(3,0.8)$ \\
$A_{9}$ & $(4,0.5) ;(5,0.5)$ & $(2,0.4) ;(3,0.6)$ & $(2,0.6) ;(3,0.4)$ & $(2,0.7) ;(3,0.3)$ & $(4,0.2) ;(5,0.8)$ \\
\hline
\end{tabular}

Table 2 Retailers' uncertain preference ordinal values for suppliers under different states

\begin{tabular}{cccccccccc}
\hline & $A_{1}$ & $A_{2}$ & $A_{3}$ & $A_{4}$ & $A_{5}$ & $A_{6}$ & $A_{7}$ & $A_{8}$ & $A_{9}$ \\
\hline$B_{1}$ & $(2,0.3) ;$ & $(1,0.3) ;$ & $(-, 0.2) ;$ & $(-, 0.4) ;$ & $(2,0.5) ;$ & $(3,0.3) ;$ & $(-, 0.2) ;$ & $(-, 0.3) ;$ & $(5,0.2) ;$ \\
& $(3,0.4) ;$ & $(2,0.3) ;$ & $(7,0.3) ;$ & $(-, 0.3) ;$ & $(3,0.4) ;$ & $(3,0.4) ;$ & $(6,0.3) ;$ & $(4,0.4) ;$ & $(7,0.4) ;$ \\
& $(-, 0.3)$ & $(3,0.4)$ & $(8,0.5)$ & $(8,0.3)$ & $(-, 0.1)$ & $(-, 0.3)$ & $(8,0.5)$ & $(7,0.3)$ & $(9,0.4)$ \\
\hline$B_{2}$ & $(3,0.3) ;$ & $(2,0.3) ;$ & $(-, 0.2) ;$ & $(4,0.4) ;$ & $(5,0.5) ;$ & $(2,0.3) ;$ & $(2,0.2) ;$ & $(7,0.3) ;$ & $(2,0.2) ;$ \\
& $(4,0.4) ;$ & $(3,0.3) ;$ & $(6,0.3) ;$ & $(5,0.3) ;$ & $(6,0.4) ;$ & $(3,0.4) ;$ & $(3,0.3) ;$ & $(8,0.4) ;$ & $(3,0.4) ;$ \\
& $(5,0.3)$ & $(5,0.4)$ & $(7,0.5)$ & $(6,0.3)$ & $(9,0.1)$ & $(-, 0.3)$ & $(-, 0.5)$ & $(9,0.3)$ & $(-, 0.4)$ \\
\hline$B_{3}$ & $(2,0.3) ;$ & $(-, 0.3) ;$ & $(-, 0.2) ;$ & $(2,0.4) ;$ & $(3,0.5) ;$ & $(5,0.3) ;$ & $(-, 0.2) ;$ & $(-, 0.3) ;$ & $(-, 0.2) ;$ \\
& $(3,0.4) ;$ & $(7,0.3) ;$ & $(-, 0.3) ;$ & $(3,0.3) ;$ & $(3,0.4) ;$ & $(7,0.4) ;$ & $(4,0.3) ;$ & $(4,0.4) ;$ & $(6,0.4) ;$ \\
& $(-, 0.3)$ & $(8,0.4)$ & $(8,0.5)$ & $(4,0.3)$ & $(-, 0.1)$ & $(9,0.3)$ & $(7,0.5)$ & $(7,0.3)$ & $(8,0.4)$ \\
\hline$B_{4}$ & $(5,0.3) ;$ & $(-, 0.3) ;$ & $(-, 0.2) ;$ & $(3,0.4) ;$ & $(1,0.5) ;$ & $(2,0.3) ;$ & $(-, 0.2) ;$ & $(-, 0.3) ;$ & $(1,0.2) ;$ \\
& $(7,0.4) ;$ & $(4,0.3) ;$ & $(6,0.3) ;$ & $(3,0.3) ;$ & $(2,0.4) ;$ & $(3,0.4) ;$ & $(-, 0.3) ;$ & $(7,0.4) ;$ & $(2,0.4) ;$ \\
& $(9,0.3)$ & $(7,0.4)$ & $(8,0.5)$ & $(-, 0.3)$ & $(3,0.1)$ & $(-, 0.3)$ & $(8,0.5)$ & $(8,0.3)$ & $(3,0.4)$ \\
\hline$B_{5}$ & $(1,0.3) ;$ & $(-, 0.3) ;$ & $(-, 0.2) ;$ & $(5,0.4) ;$ & $(-, 0.5) ;$ & $(2,0.3) ;$ & $(3,0.2) ;$ & $(-, 0.3) ;$ & $(1,0.2) ;$ \\
& $(2,0.4) ;$ & $(7,0.3) ;$ & $(6,0.3) ;$ & $(7,0.3) ;$ & $(-, 0.4) ;$ & $(3,0.4) ;$ & $(3,0.3) ;$ & $(4,0.4) ;$ & $(2,0.4) ;$ \\
& $(3,0.3)$ & $(8,0.4)$ & $(8,0.5)$ & $(9,0.3)$ & $(8,0.1)$ & $(-, 0.3)$ & $(-, 0.5)$ & $(7,0.3)$ & $(3,0.4)$ \\
\hline
\end{tabular}

The method proposed in this paper is used to find a fully satisfactory solution for both parties. The process is briefly described in the following steps:

1) Assuming that a five-level ranking system $\left\{H_{1}, H_{2}, H_{3}, H_{4}, H_{5}\right\}$ is used, based on Equation (1), we know that suppliers' standard ranking values are $\left\{Y_{1}, Y_{2}, Y_{3}, Y_{4}, Y_{5}\right\}=\{1,3,5,7,9\}$, and the retailers' standard ranking values are $\left\{Y_{1}, Y_{2}, Y_{3}, Y_{4}, Y_{5}\right\}=\{1,2,3,4,5\}$.

2) To determine the data relationship between preference order information and standard ranking values, in accordance with Equations $(3) \sim(5)$, we transform the suppliers' and retailers' preference ordinal values under different uncertain states into rank belief degree information. 
3) From Equations $(6) \sim(12)$, we obtain two-sided average satisfaction degrees, as shown in Table 3.

4) From Equations (13) (14), by employing satisfaction degrees, we find the following twosided matching solution: $A_{1}$ and $B_{5}, A_{2}$ and $B_{2}, A_{4}$ and $B_{3}, A_{5}$ and $B_{1}, A_{6}$ and $B_{4}$; that is, $A_{1} \leftrightarrow B_{5}, A_{2} \leftrightarrow B_{2}, A_{4} \leftrightarrow B_{3}, A_{5} \leftrightarrow B_{1}, A_{6} \leftrightarrow B_{4}$.

Table 3 Retailer and supplier average satisfaction degrees

\begin{tabular}{llllllllll}
\hline & $A_{1}$ & $A_{2}$ & $A_{3}$ & $A_{4}$ & $A_{5}$ & $A_{6}$ & $A_{7}$ & $A_{8}$ & $A_{9}$ \\
\hline$B_{1}$ & 0.769 & 0.756 & 0.311 & 0.384 & 0.755 & 0.744 & 0.433 & 0.581 & 0.200 \\
$B_{2}$ & 0.574 & 0.683 & 0.514 & 0.429 & 0.358 & 0.658 & 0.653 & 0.369 & 0.615 \\
$B_{3}$ & 0.737 & 0.569 & 0.326 & 0.654 & 0.666 & 0.465 & 0.626 & 0.371 & 0.480 \\
$B_{4}$ & 0.244 & 0.702 & 0.488 & 0.602 & 0.786 & 0.826 & 0.508 & 0.500 & 0.741 \\
$B_{5}$ & 0.800 & 0.390 & 0.270 & 0.156 & 0.514 & 0.684 & 0.613 & 0.523 & 0.675 \\
\hline
\end{tabular}

\section{Conclusion}

Research on two-sided matching decision-making with uncertainty under various states is a beneficial addition to the existing body of matching decision-making theory, as it has considerable application value and research significance. Using the foundation of ER, this paper proposed tools for solving two-sided matching problems with uncertain preference ordinal values under multiple states. This reasoning is simple and logical, and the method was verified as feasible through an illustration. The proposed method is suitable for solving two-sided matching problems with uncertainty under different states in fields such as economic management and human resources. However, more questions remain, such as how we may obtain the occurrence probabilities of states and so forth.

\section{References}

[1] Gale D, Shapley L. College admissions and the stability of marriage. American Mathematical Monthly, 1962, 69(1): 9-15.

[2] Kojima F. School choice: Impossibilities for affirmative action. Games and Economic Behavior, 2012, 75(2): 685-693.

[3] Ehlers L, Hafalir I E, Yenmez M B, et al. School choice with controlled choice constraints: Hard bounds versus soft bounds. Journal of Economic Theory, 2014, 153: 648-683.

[4] Mindruta D, Moeen M, Agarwal R. A two-sided matching approach for partner selection and assessing complementarities in partners' attributes in inter-firm alliances. HEC Paris Research Paper No. SPE-20141068, 2014.

[5] Huang D K, Chiu H N. Yeh R H, et al. A fuzzy multi-criteria decision making approach for solving a bi-objective personnel assignment problem. Computers \& Industrial Engineering, 2009, 56(1): 1-10.

[6] Lin S Y, Horng S J, Kao T W, et al. Solving the bi-objective personnel assignment problem using particle swarm optimization. Applied Soft Computing, 2012, 12(9): 2840-2845.

[7] Klerkx L L. Matching demand and supply in the agricultural knowledge infrastructure: Experiences with innovation intermediaries. Food Policy, 2008, 33(3): 260-276.

[8] Alam N, Lee Ng S. Banking mergers - An application of matching strategy. Review of Accounting and Finance, 2014, 13(1): 2-23.

[9] Anjos F, Drexler A. Inter-company matching and the supply of informed capital. Journal of Economic Behavior \& Organization, 2015, 111: 119-136. 
[10] Chen J, Song K. Two-sided matching in the loan market. International Journal of Industrial Organization, 2013, 31(2): 145-152.

[11] Terviö M. The difference that CEOs make: An assignment model approach. The American Economic Review, 2008, 98(3): 642-668.

[12] Lippi F, Schivardi F. Corporate control and executive selection. Quantitative economics, 2014, 5(2): 417456.

[13] Poppo L, Zhou K Z. Managing contracts for fairness in buyer-supplier exchanges. Strategic Management Journal, 2014, 35(10): 1508-1527.

[14] Zhou K Z, Zhang Q, Sheng S, et al. Are relational ties always good for knowledge acquisition? Buyersupplier exchanges in China. Journal of Operations Management, 2014, 32(3): 88-98.

[15] Mindruta D. Value creation in university-firm research collaborations: A matching approach. Strategic Management Journal, 2013, 34(6): 644-665.

[16] Roth A E. Two-sided matching with incomplete information about others' preferences. Games and Economic Behavior, 1989, 1(2): 191-209.

[17] Chade H. Matching with noise and the acceptance curse. Journal of Economic Theory, 2006, 129(1): 81-113.

[18] Ehlers L, Massó J. Incomplete information and singleton cores in matching markets. Journal of Economic Theory, 2007, 136(1): 587-600.

[19] Hoppe H C, Moldovanu B, Sela A. The theory of assortative matching based on costly signals. The Review of Economic Studies, 2009, 76(1): 253-281.

[20] Chakraborty A, Citanna A, Ostrovsky M. Two-sided matching with interdependent values. Journal of Economic Theory, 2010, 145(1): 85-105.

[21] Rastegari B, Condon A, Immorlica N, et al. Two-sided matching with partial information. Proceedings of the Fourteenth ACM Conference on Electronic Commerce, ACM, 2013: 733-750.

[22] Chade H, Lewis G, Smith L. Student portfolios and the college admissions problem. The Review of Economic Studies, 2014, 81(3): 971-1002.

[23] Liu Q, Mailath G J, Postlewaite A, et al. Stable matching with incomplete information. Econometrica, 2014, 82(2): 541-587.

[24] Chen X, Fan Z P, Li Y H. A fuzzy multi- objective decision making method for two-sided matching of supply and demand in IT service. Chinese Journal of Management, 2011, 32(2): 297-302.

[25] Yue Q. decision method for the two-sided matching with uncertain preference ordinal information based on cumulative prospect theory. Journal of Systems Science and Mathematical Sciences, 2013, 33(9): 1061-1070.

[26] Yue Q. Indifference interval multiple criteria matching decision method. Journal of Systems Engineering, 2014, 29(1): 41-47.

[27] Yue Q. Decision method for two-sided matching based on incomplete order relation information. Journal of Zhejiang University (Science Edition), 2014, 41(5): 523-527.

[28] Liang H M, Jiang Y P. Method for two-sided matching decision-making based on the weak preference ordering information. Journal of Systems Engineering, 2014, 29(2): 153-159.

[29] Liang H M, Jiang Y P. Decision-making method on second-hand house combination matching. Systems Engineering — Theory \& Practice, 2015, 35(2): 358-367.

[30] Zhang L L, Hu X P. "Team-working object" matching model based on human capital competition for optimal first structure. Journal of Industrial Engineering and Engineering Management, 2015, 29(1): 1-7.

[31] Chen S Q, Wang Y M, Shi H L. A dynamic matching decision-making method based on ordinal deviation fusion degrees. Operations Research and Management Science, 2014, 23(1): 59-65.

[32] Chen S Q, Wang Y M, Shi H L. Data fusion method for multi-arrtibute matching decision-making with rank belief degrees. Journal of Systems Engineering, 2015, 30(1): 25-33.

[33] Wang Y M, Yang J B, Xu D L. Environmental impact assessment using the evidential reasoning approach. European Journal of Operational Research, 2006, 174(3): 1885-1913.

[34] Yang J B, Wang Y M, Xu D L, et al. The evidential reasoning approach for MADA under both probabilistic and fuzzy uncertainties. European Journal of Operational Research, 2006, 171(1): 309-343.

[35] Dempster A P. Upper and lower probabilities induced by a multivalued mapping. Annals of Mathematical Statistics, 1967, 38(2): 325-339.

[36] Shafer G. A mathematical theory of evidence. Princeton: Princeton University Press, 1976. 